\title{
Efficiency Variations in Electrically Small, Meander Line RFID Antennas
}

\author{
Amir Galehdar, David V. Thiel, Steven G. O'Keefe and Simon P. Kingsley \\ Centre for Wireless Monitoring \& Applications \\ Griffith School of Engineering, Griffith University - Nathan \\ Queensland, 4111, Australia \\ E-mail: s2145033@student.griffith.edu.au, d.thiel@griffith.edu.au
}

\begin{abstract}
Not all meander line dipole antennas (MLDAs) plotted on the same grid necessarily have similar characteristics. MLDAs can be optimized for best efficiency, lowest resonant frequency or highest impedance. Different structures were investigated using a $5 \times 10$ array of rectangular grid points in $4 \times 9 \mathrm{~mm}^{2}$ area and, all symmetrical MLDA that incorporate all grids points were modeled to determine the resonant frequency and efficiency. Based on the NEC simulation results for all possible family members, the range in resonant frequencies was 3.51 to $4.71 \mathrm{GHz}$ and the range in efficiencies was $58.48 \%$ to $83.17 \%$. The most efficient structure and the structure with the lowest resonant frequency were determined from the entire population of 1072 different antenna structures. The most efficient antennas have identical structures close to the feed point and all have an efficiency of greater than $83 \%$.
\end{abstract}

Key words: RFID, antenna efficiency, NEC, Hilbert antenna, Koch antenna.

\section{Introduction}

The concept of radio frequency identification (RFID) can be traced back to 1948 [1], however the recent increase in commercial interest has resulted in new challenges, in particular read-range. This is defined as "the maximum distance at which RFID reader can detect the backscattered signal from the tag" [2]. The read range can be calculated from a modified Friis free-space formula where antenna efficiency is an important design constraint. Another design limitation is the antenna size, and so engineers continue to seek the most efficient antenna structure for a given area.

A number of previous studies concentrated on a performance comparison between a meander antenna and Hilbert antenna [3] or Koch fractal antenna [4]. In this paper the efficiency of meander structures with the same physical area have been studied. These antennas were simulated by NEC and their efficiencies at the resonant frequencies were determined. These results are compared to find the antenna design characteristics with the highest efficiency and those with the lowest resonant frequency (defined as the frequency where the input impedance is purely real).

\section{Method:}

In order to categorize the meanders, some restrictions were set to limit the number of possible structures. The track grid separation was set at $1 \mathrm{~mm}$ and the antenna half-area was set at $4 \times 4 \mathrm{~mm}^{2}$ area with tracks mapped to $5 \times 5$ grid points (Fig 1.a). The dipole was centre fed along the line of symmetry. That is one half-area was the mirror image of the other half-area. By connecting all grid points, a meander line with the half length of $24 \mathrm{~mm}$ is produced (Fig 1.b). By connecting two half areas together through a $1 \mathrm{~mm}$ 
bridge (and feed) a dipole meander antenna is formed so the final antenna (Fig 2) occupies $4 \times 9 \mathrm{~mm}^{2}$ and has total length of $49 \mathrm{~mm}$.

In order to find all possible combinations, the 25 grid points in the half area were numbered and the following construction rules were applied:

- The meander line starts from one of the points on the line of symmetry as it needs to be connected to other cell.

- The meander line can pass through each point once only.

- Only three feed points on the line of symmetry were considered because the antenna built from the other two points produce repeated patterns. Thus points 1,2 and 3 were studied as starting points.

All possible permutations joining these 25 points that can generate a meander line antenna are produced by following these rules and applying tree algorithm [5]. Starting from point 1 , there are 824 meander structures. There are no possible meander structures starting from point two. Point three is the starting point for 496 different combinations but half of these combinations are inverted versions of others so the maximum number is reduced to 248. The total number of possible antennas in this meander family was 1072 . All members of the family were constructed and submitted to NEC modeling with $0.1 \mathrm{~mm}$ copper (conductivity $5.8 \times 10^{7} \mathrm{~S} / \mathrm{m}$ ) wire. The efficiency at resonant frequency of all antennas was determined using the current segment method outlined in [6].

\section{Results:}

The family of 1072 meander antennas was found to have resonant frequencies in the range $3.51 \mathrm{GHz}$ to $4.71 \mathrm{GHz}$. Best and Morrow [3] devised a meander structure with a low resonant frequency for a given area. An identical structure (see Fig 3(a)) was found to resonate at $3.83 \mathrm{GHz}$. The structure with the lowest resonant frequency of 3.51GHz is shown in Fig 3(b).

The antenna efficiency at the resonant frequency of all the family members was found to vary from $58.48 \%$ to $83.17 \%$. Additionally, the efficiency at resonant frequency of the eight most efficient structures is plotted in Fig 4 together with their resonant frequencies. It is evident that the efficiency difference is less than $1 \%$ difference; however, there is more that $100 \mathrm{MHz}$ difference in resonant frequencies. The most efficient structure with $83.17 \%$ efficiency at $4.68 \mathrm{GHz}$ resonant frequency is shown in Fig 5. The antenna efficiencies over the range of 3 to $5 \mathrm{GHz}$ are plotted in Fig 6 . The trend lines are almost identical.

\section{Conclusions}

In this paper all the symmetrical RFID dipole meander antennas in the given area have been studied. There are 1072 different combinations of meander antenna with the track separation distance of $1 \mathrm{~mm}$ in a $4 \times 9 \mathrm{~mm}^{2}$ area. All the family members were simulated in NEC and their resonant frequencies were measured and varied from 3.51 to 4.71 $\mathrm{GHz}$. The efficiencies were measured over the 3 to $5 \mathrm{GHz}$ frequency band. From these results the structure with the lowest resonant frequency $3.51 \mathrm{GHz}$ and the structure with the highest efficiency $83.17 \%$ at resonant frequency were determined. This approach allows MLDAs to be designed for either maximum efficiency or minimum size. These properties can be important when designing antennas that must be electrically very small, such as RFID tags. 


\section{References:}

[1] H. Stockman, "Communication by means of reflected power," Proc. IRE, pp. 1196-1204, Oct. 1948.

[2] K.V. Seshagiri Rao, P. V. Nikitin, and S. F. Lam, "Antenna design for UHF RFID tags: A review and a practical application," IEEE Trans. Antennas Propag., vol. 53, pp. 3870-3876, 2005.

[3] Steve R. Best and Jarrett D. Morrow, "The effectiveness of space-filling fractal geometry in lowering resonant frequency," IEEE Antenna and Wireless Propag., vol. 1, pp. 112-115, 2002.

[4] Steve R. Best, "On the performance propertied of the Koch fractal and other bent wire monopoles," IEEE Trans. Antennas Propag., vol. 51, pp. 1292-1300, 2003.

[5] Mikhail J. Atallah, Algorithms and Theory of Computation Handbook, 1st edition, CRC Press, 1999.

[6] Amir Galehdar, David V. Thiel and Steven G. O'Keefe, "Antenna Efficiency Calculations for Electrically small, RFID antennas," IEEE Antenna and Wireless Propag., (in press), 2007.

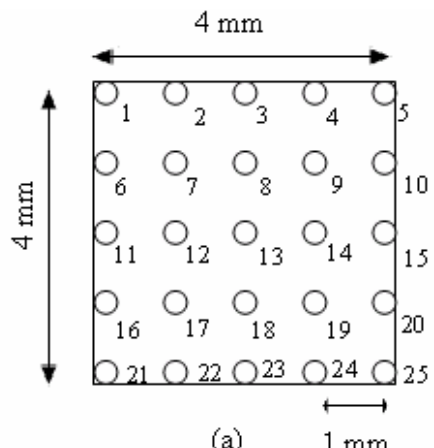

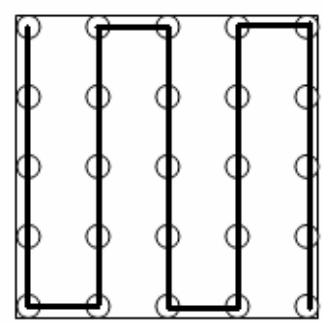

(b)

Fig 1: (a) Grid points used on a $4 \times 4 \mathrm{~mm}^{2}$; (b) One example of a meander line cell produced by connecting all grid points.

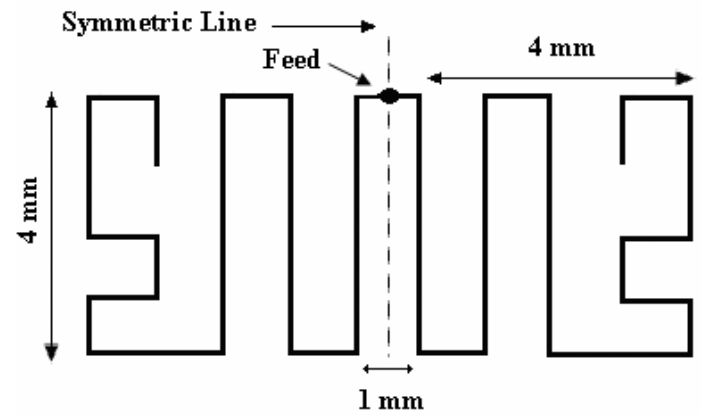

Fig 2: A dipole RFID meander antenna produced by Fig 1 (b) cells. 


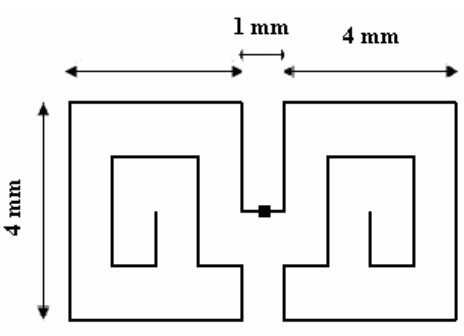

(a)

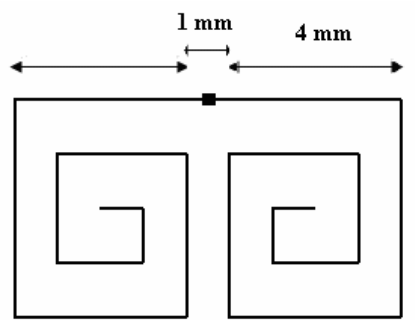

(b)

Fig 3: (a) The antenna structure proposed in [3], (b) Lowest frequency antenna structure.

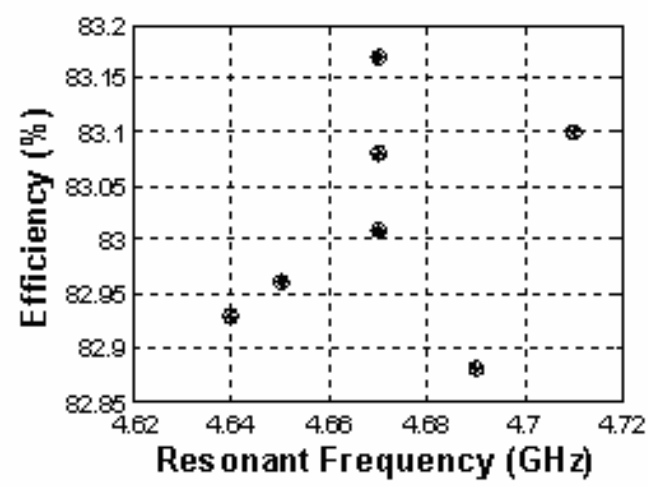

Fig 4: The efficiency verses resonant frequency of the eight most efficient structures.

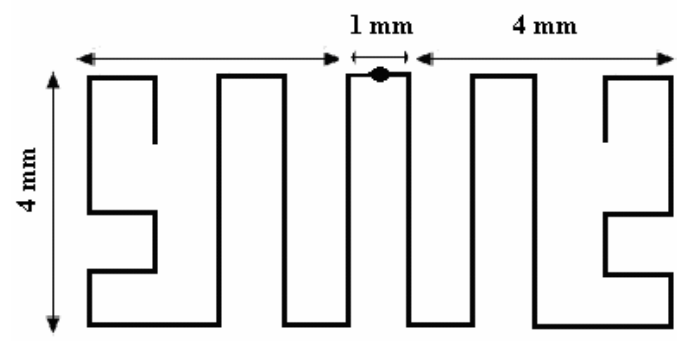

Fig 5: The structure of the most efficient antenna in a given area.

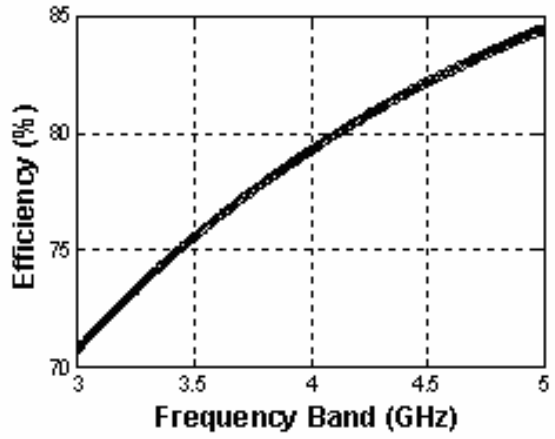

Fig 6: The efficiency verses frequency for the eight most efficient structures near their resonance. 